\title{
Pathologic analysis and prognosis observation of 81 cases of pure GGO after surgical resection.
}

\author{
Ren Zhi-peng\#, Hou Xiao-bin\#, Zhang Lian-bin, Liu Yi, Wang Bin, Wen Jia-xin Chu Xiang-yang* \\ Department of Thoracic Surgery, Chinese PLA (The People's Liberation Army) General Hospital, Beijing, PR China \\ \#These authors contributed equally to this work
}

\begin{abstract}
Objective: To observe the malignant proportion and prognosis of pure ground-glass opacity (pGGO). Method: All cases of pGGO were surgical resected from January 2010 to May 2013 in The People's Liberation Army General Hospital. At the same time, comply with the requirements for the following three cases included in this study: (1) on the imaging performance for pGGO; (2) postoperative followup time more than 2 years; (3) to eliminate multiple pGGO and then analyse the postoperative pathologic results, prognosis and observation.

Results: Participants included 81 cases of pGGO, 62 cases were malignant tumors (76.5\%), 11 patients of AAH (13.6\%), 8 cases of benign disease (9.9\%). 57 cases in the follow up study of preoperative pGGO were steady, and in this subgroup of pGGO pathology: 43 cases of malignant tumors (75.4\%), including 39 cases of AIS, 3 case of MIA, 1 case of adenocarcinoma; 9 patients of AAH (15.8\%), 5 cases of benign lesions $(8.8 \%)$. In 14 cases of pGGO before surgery, lesions or the density increases during the observation period. In this subgroup nine cases showed cancer pathology with malignant rate of $92.9 \%$. The median postoperative follow-up time was 34.7 months. 1 patient during follow-up emerged new lesions in a different lobe, still pGGO, and was still in follow-up observation with stable disease, and no surgery. All the remaining patients were found no local recurrence and distant metastasis, and no patient died.

Conclusions: The pGGO that follow up with no change should be relatively positive for surgery, and the pGGO that follow up with lesions especially the density increased in the observation process is necessary of surgical treatment.
\end{abstract}

Keywords: Ground-glass opacity, GGO, Surgery, Pathology, Prognosis.

Accepted on March 16, 2018

\section{Introduction}

The application of CT, especially low-dose CT on lung cancer screening instead of chest radiography, brings the mortality of lung cancer down about $20 \%$. Imaging diagnosis of groundglass opacity becomes more and more common [1]. GGOs' detecting rate of the screening patients is among $0.2 \%$ to $0.5 \%$ [2]. In 1996, Austin described the detected GGO on HRCT: lung tissue density softly increased, whereas bronchus and blood vessel bundle were still visible [3]. Single GGO lesions can be diagnosed variously, such as infection or inflammation, fibrosis, AAH, adenocarcinoma in situ or invasive adenocarcinoma [4]. The advantage of thoracic surgeons treating GGO lesions is that they can give the patients diagnose and treatment simultaneously. As GGO lesions grow slow, the operation timing is not explicitly stipulated [5]. According to 2014 NCCN Guideline of Lung Cancer Screening, surgical resection will be in consideration if pure GGO increases in size or density. If pure GGO has no change in the follow-up observation, then we can stay observing. While it is also reported that even if the pure GGO is without any change, it gets malignant at a high rate [6]. Clinical research of GGO also has dilemma because of the number limitation of GGO cases and the long-term follow-up observation as GGO lesions grows slow [7]. The purposes of our study are to analyse the postoperative pathology of pure GGO especially the no-change cases during follow-up observation and to observe the prognosis of the cases that have been observed over 2 years.

\section{Materials and Methods}

\section{Patients}

The data used in this study were collected from the records of patients who underwent surgical resection for pure GGOs in Department of Thoracic Surgery of the People's Liberation Army General Hospital during January 2010 to May 2013. The proportion of GGOs in a nodule was measured in the same way as that reported elsewhere [8]. In brief, the images were photographed with a window level of $600 \mathrm{H}$ and a window 
width of 2,000 $\mathrm{H}$ as the lung window. GGO lesions were classified as pure GGO lesions only if they had a $100 \% \mathrm{GGO}$ pro-portion. Enrolled patients have to meet the following 4 conditions: (1) pure GGOs were detected with HRCT, (2) postoperative follow-up at least 2 years and (3) multiple pure GGOs were excluded.

\section{Surgical indication}

(1) Growth of the nodules or of newly formed solid components in pure GGOs was detected;

(2) In the surgeons' judgment based on their experience with pure GGOs, initial CT findings strongly suggested cancer, in which case surgical resection was performed without a period of observation.

(3) Patients with pure GGOs, if no change was observed after minimum 6-month follow-up period.

\section{Surgical procedures}

During the study periods, there was a guideline for the extent of surgical resection. If the preoperative diagnosis was not confirmed, wedge resection by use of Video- Assisted Thoracic Surgery (VATS) (aided by CT-guided hook-wire localization in some cases) was done first, and the tissue was sent to the pathology laboratory for a frozen section. If the tissue was malignant, anatomic resection and mediastinal node dissection were usually performed, whereas limited resection including wide wedge resection or segmentectomy was pursued in selected cases.

\section{Results}

\section{Patients}

81 patients were enrolled in this study group, 53 were female, and the average age was $58.3 \mathrm{y}$ old (range, 39 to $75 \mathrm{y}$ old). 57 patients experienced no change of their pure GGO nodules during at least 6-month follow-up, which met the major indication for operation, and 14 patients underwent surgical resection because of growth of the lesions or new development of solid lesions during follow-up. 10 patients underwent surgical resection immediately after diagnosis with pure GGOs.

\section{Pathology}

Within the 81 patients, pathologic types of malignant tumors pGGOs were in 62 patients $(76.5 \%)$ included AIS in 52 patients ( $3 \mathrm{~mm}$ in diameter minimum, Figure 1 ), MIA in 6 patients, and invasive adenocarcinoma in 4 patients (Figures 2 and 3) without lymph node metastasis, AAH in 11 patients $(13.6 \%)$, and 8 patients $(9.9 \%)$ with benign lesions.

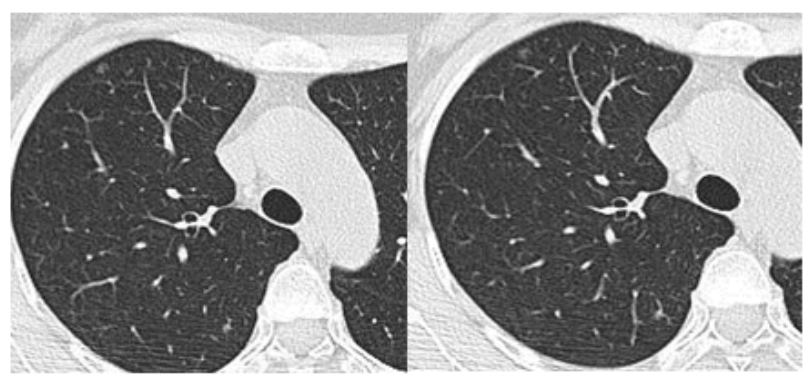

Figure 1. Tiny nodules at anterior segment of right upper lobe, $3 \mathrm{~mm}$, pure GGO, wedge resection by use of Video-Assisted Thoracic Surgery (VATS) (aided by CT-guided hook-wire localization), being diagnosed as adenocarcinoma in situ.

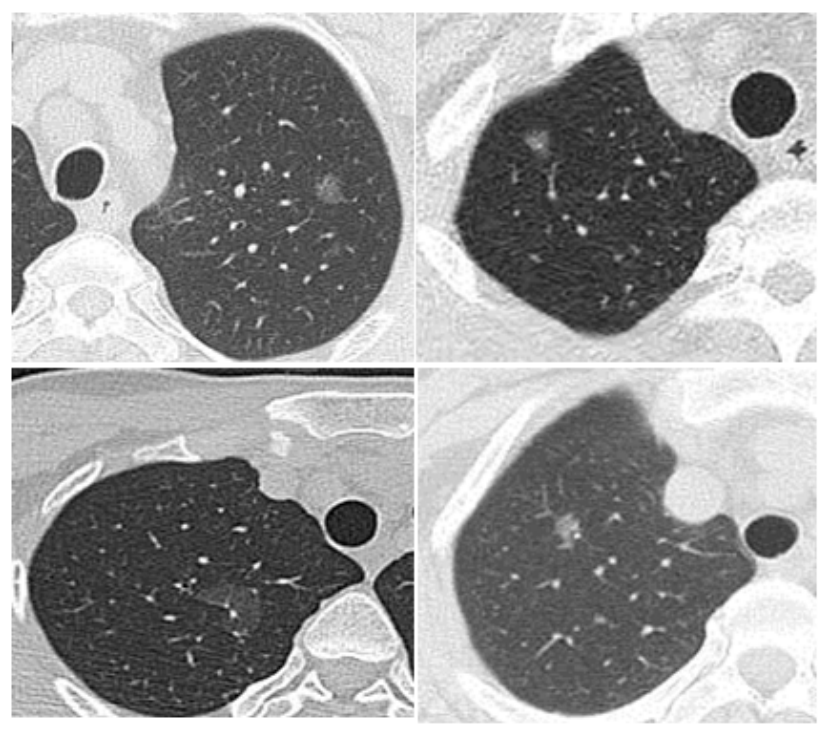

Figure 2. 4 cases of MIA on CT.

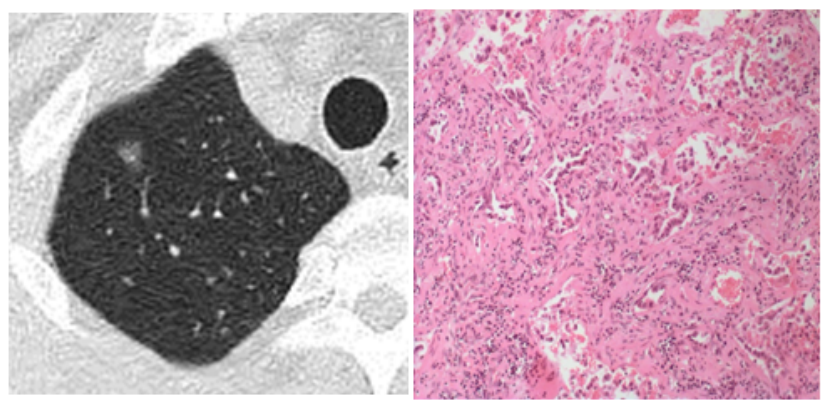

Figure 3. Pathology comparison on CT of one of the four MIAs.

\section{Surgical procedures}

34 patients were underwent the scheduled operations aided by CT-guided hook-wire localization successfully. The types of operations performed in this group included pulmonary lobectomy and lymph node dissection, wedge resection and segmentectomy, and they were performed on 53 patients, 4 patients, and 5 patients, respectively. 


\section{No-change group in all pGOOs}

There were 57 patients in this no-change group. The average time period from detection to operation was 391.6 days, and the time periods from detection to operation were 6 to 12 months in 21 patients $(36.8 \%), 12$ to 24 months in 33 patients $(57.9 \%)$, and longer than 24 months in 3 patients $(5.3 \%)$. The median size of the tumors was $1.2 \mathrm{~cm}$ (range, 0.3 to $2 \mathrm{~cm}$ ), and the tumor sizes were smaller than $1 \mathrm{~cm}$ in 24 patients $(42.1 \%)$ and 1 to $2 \mathrm{~cm}$ in 33 patients $(57.9 \%)$. Postoperative pathologic types of malignant tumors pGGOs in 43 patients included AIS in 39 patients, MIA in 3 patient and invasive adenocarcinoma in 1 patient; pathologic types of AAH in 9 patients $(15.8 \%)$ and of benign lesions in $5(8.8 \%)$ Figure 4.
A

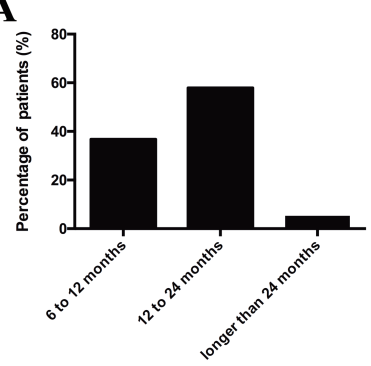

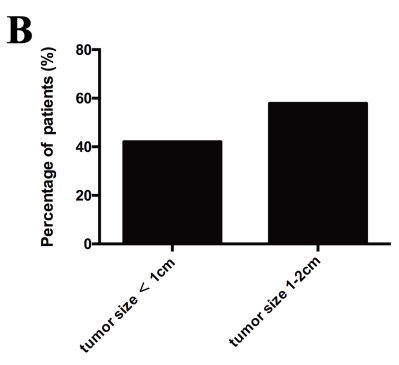

Figure 4. The tumor size and treatment time period in No-change group.

\section{Lesions increasing in size or density group}

There were 13 out of 14 patients in this group diagnosed malignant tumors pGGOs. In the observation period (95 425 d), lesions increased the most in 9 patients. Postoperative pathologic: inflammatory lesion in 1 patient, AIS in 6 patients, MIA in 2 patients. And in the observation period (125 $664 \mathrm{~d}$ ), lesion density increased in 5 patients, including 1 AIS and 4 adenocarcinomas.

\section{Direct surgical group}

This group consisted of 10 patients, 7 of whom were malignant tumors pGGOs, including AIS in 5 patients, and MIA in 2 patients; AAH in 2 patients and pulmonary lobectomy in 1 patient respectively.

\section{Prognosis}

Postoperative follow-up time was 13.7 months. One patient got new lesions that were stable in the other pulmonary lobe and still pure GGO during the follow-up. He got no surgery yet. No local recurrence and distant metastasis found in the rest patients and no death.

\section{Comment}

According to 2014 version 1.2014 NCCN Guideline of Lung Cancer Screening, surgical resection will be in consideration if pure GGO increases in size or density during observation. If pure GGO has no change in the follow-up observation, then we can stay observing. While it is also reported that even if the pure GGO is without any change, it gets malignant at a high rate $[6,9,10]$. The authors don't think that the guideline provide a satisficing answers to the operation time. It is also reported that the pGGO intraoperative localization of the lesions that are far from visceral pleura is problematic, especially hard to achieve in the video-assisted thoracoscopic surgery. In such cases, it's suggested to observe in the follow-up. Zaman et al. meta-analysis showed that the success of CT-guided hook-wire technique to the video-assisted thoracoscopic pulmonary nodules ranged from $58 \%$ to $97 \%$ [11]. This technique effectively solved the most intraoperative localization issue. In our hospital, we have our own intraoperative CT equipment. In this study, CT guided hook-wire localization of lesions succeeded in 34 patients, and lesions were excised precisely, none failed. VATS were undergone as soon as lesions were located by hook wire, without delay and no cases of opening chest during operation. The most common complications were pneumothorax and pulmonary hemorrhage of a very small amount by CT screening, while there would be no adverse effects to the patients because of the immediate VATS after localization. The application of CT-guided hook-wire localization could effectively solve the localization of tiny nodules of pGGOs and also it is a pre-condition for the minimally invasive resection. There is still limitation of hookwire localization for some lesions that are near the mediastinal large blood vessels and deep in the lung parenchyma. So for the no change pGGOs in the follow-up, we suggest to carry out surgical treatment. For the lesions increasing in size or density, surgical treatment is more necessary and especially minimally invasive. For the lesions that are difficult to locate, hook-wire localization is a good choice. As the imaging manifestations of pGGO lung cancer develop slowly, we shall be very careful for the elderly patients and the patients who have high surgery risks.

Postoperative prognosis of pGGO lesions is super good. Park et al. followed up 58 patients of malignant pGGO in two years, and found no local recurrence or distant metastasis [12]. In Kondo et al.'s and Ichiki et al.'s researches, 5 y survival rate after malignant pGGO surgery was as high as $100 \%[13,14]$. The follow-up period in our study was not long enough to reflect real prognosis. And we think a $5 \mathrm{y}$ period is not a sufficient follow-up time to see the survival rate. Many pGGO lesions did not cause any death with no intervention in 5 or even longer years. So we shall extend the postoperative followup period for the future pGGO prognosis study. In our study, we will do further follow-up work, and expand the number of patients to provide more evidence for the further pGGO study.

In conclusion, this study showed the pathology of persistent stable pGGO postoperatively, which confirmed the $75.4 \%$ chance of the cancer diagnosis including AIS, MIA, or invasive adenocarcinoma, and $15.8 \%$ for $\mathrm{AAH}, 8.8 \%$ for benign lesions. While the lesions that increased in size or density got malignant at even high rate of $92.9 \%$. Therefore, it's better to perform surgical resection to the no-change patients, and even more necessary to perform surgical resection to the patients whose lesions increasing in size or density. 


\section{Conflict of Interests}

The authors confirm that this article content has no conflict of interest.

\section{References}

1. Aberle DR, Adams AM, Berg CD. Reduced lung-cancer mortality with low-dose computed tomographic screening. National lung screening trial research team. N Engl J Med 2011; 365: 395-409.

2. Henschke CI, Shaham D, Yankelevitz DF. CT screening for lung cancer: significance of diagnoses in its baseline cycle. Clin Imaging 2006; 30: 11-15.

3. Austin JH, Muller NL, Friedman PJ. Glossary of terms for CT of the lungs: recommendations of the Nomenclature Committee of the Fleischner Society. Radiology 1996; 200: 327-331.

4. Godoy MC, Naidich DP. Overview and strategic management of subsolid pulmonary nodules. J Thorac Imaging 2012; 27: 240-248.

5. Boris S, Garrett W. Surgical therapy of ground-glass opacities. Sem Diagn Pathol 2014; 31: 289-292.

6. Cho S, Yang H, Kim K, Jheon S. Pathology and prognosis of persistent stable pure ground-glass opacity nodules after surgical resection. Ann Thorac Surg 2013; 96: 1190-1195.

7. Junji Y. Management of the peripheral small ground-glass opacities. Thorac Surg Clin 2007; 17: 191-201.

8. Suzuki K, Asamura H, Kusumoto M, Kondo H, Tsuchiya R. Early peripheral lung cancer: prognostic significance of ground glass opacity on thin-section computed tomographic scan. Ann Thorac Surg 2002; 74: 1635-1639.
9. Duann C, Hung J, Hsu P. Surgical outcomes in lung cancer presenting as ground-glass opacities of $3 \mathrm{~cm}$ or less: A review of 5 years of experience. J Chinese Med Assoc 2013; 76: 693-697.

10. Ken K, Masahiko H, Hideoki Y. Natural history of pure ground-glass opacity after long-term follow-up of more than 2 years. Ann Thorac Surg 2002; 73: 386-393.

11. Zaman M, Bilal H, Woo CY, Tang A. In patients undergoing video-assisted thoracoscopic surgery excision, what is the best way to locate a subcentimetre solitary pulmonary nodule in order to achieve successful excision? Interact Cardiovasc Thorac Surg 2012; 15: 266-272.

12. Park JH, Lee KS, Kim JH, Shim YM, Kim J, Choi YS. Malignant pure pulmonary ground-glass opacity nodules: prognostic implications. Korean J Radiol 2009; 10: 12-20.

13. Kondo T, Yamada K, Noda K, Nakayama H, Kameda Y. Radiologic prognostic correlation in patients with small pulmonary adenocarcinomas. Lung Cancer 2002; 36: 49-57.

14. Ichiki Y, Hanagiri T, Baba T, So T, Ono K, Uramoto $H$. Limited pulmonary resection for peripheral small-sized adenocarcinoma of the lung. Int J Surg 2011; 9: 155.

\section{*Correspondence to}

Chu Xiang-yang

Department of Thoracic Surgery

Chinese PLA (The People's Liberation Army) General Hospital

PR China 